\title{
Building digital government strategies. Principles and practices [Construyendo estrategias de gobierno digital. Principios y prácticas]
}

\begin{abstract}
Sandoval-Almazán, R., Luna Reyes, L. F., Luna Reyes, D. E., Gil-Garcia, J. R., Puron-Cid, G., \& Picazo-Vela, S. (2017). Public Administration and Information Technology [Construyendo estrategias de gobierno digital. Principios y prácticas] (114 pp., ISBN 978-3-319-60347-6, doi 10.1007/978-3-319-60348-3). San Antonio, US: Springer International Publishing AG.
\end{abstract}

Manuel Díaz Flores*凶

Díaz Flores, M. (2018). Building digital government strategies. Principles and practices [Construyendo estrategias de gobierno digital. Principios y prácticas]. Reseña [Reseña de libro] Investigación y Ciencia de la Universidad Autónoma de Aguascalientes, 26(73), 97-98.

El Plan Nacional de Desarrollo 2013-2018 se planteó como objetivo establecer una Estrategia Digital Nacional para acelerar la inserción de México en la Sociedad de la Información y del Conocimiento. Entre los logros más relevantes reportados por el propio gobierno federal está la Ventanilla Única Nacional para los Trámites e Información del Gobierno. De este modo, este portal ha contribuido a que el número de personas que utilizan Internet para interactuar con el gobierno haya aumentado muy significativamente. Sin embargo, a medida que se hace una revisión de la incorporación de las tecnologías de la información por los gobiernos estatales y aún más en los municipios el panorama es muy diverso, ya que solamente la mitad de los ayuntamientos de México han implementado acciones relacionadas con el índice de gobierno electrónico, el cual incorpora: indicador de computadoras, de líneas telefónicas, conexión a internet, información en sitio web y su funcionalidad. Entre las limitaciones más relevantes para avanzar en este proceso se señalan la falta de capacitación de los recursos humanos, la infraestructura y el financiamiento.

Adicionalmente, los tres niveles de gobierno tienen actualmente importantes requerimientos de información para cumplir con la Ley Federal de Transparencia y Acceso a la información Pública Gubernamental, así como la rendición de cuentas. En este contexto, se publica este libro, una herramienta sumamente útil ya que es el resultado de investigaciones académicas tradicionales, de lecciones aprendidas

\footnotetext{
* Departamento de Economía, Centro de Ciencias Económicas y Administrativas, Universidad Autónoma de Aguascalientes. Avenida Universidad 940, Ciudad Universitaria, C. P. 20131, Aguascalientes, Aguascalientes, México. Correo electrónico: mdiaz@correo.uaa.mx

a través de la práctica profesional y la experiencia de consultoría. Está dirigido a los funcionarios públicos, administradores, consultores del gobierno, líderes de organizaciones no gubernamentales interesados en promover y desarrollar el gobierno digital. No da soluciones para cada situación particular, porque cada problema es diferente en su contexto, es más una

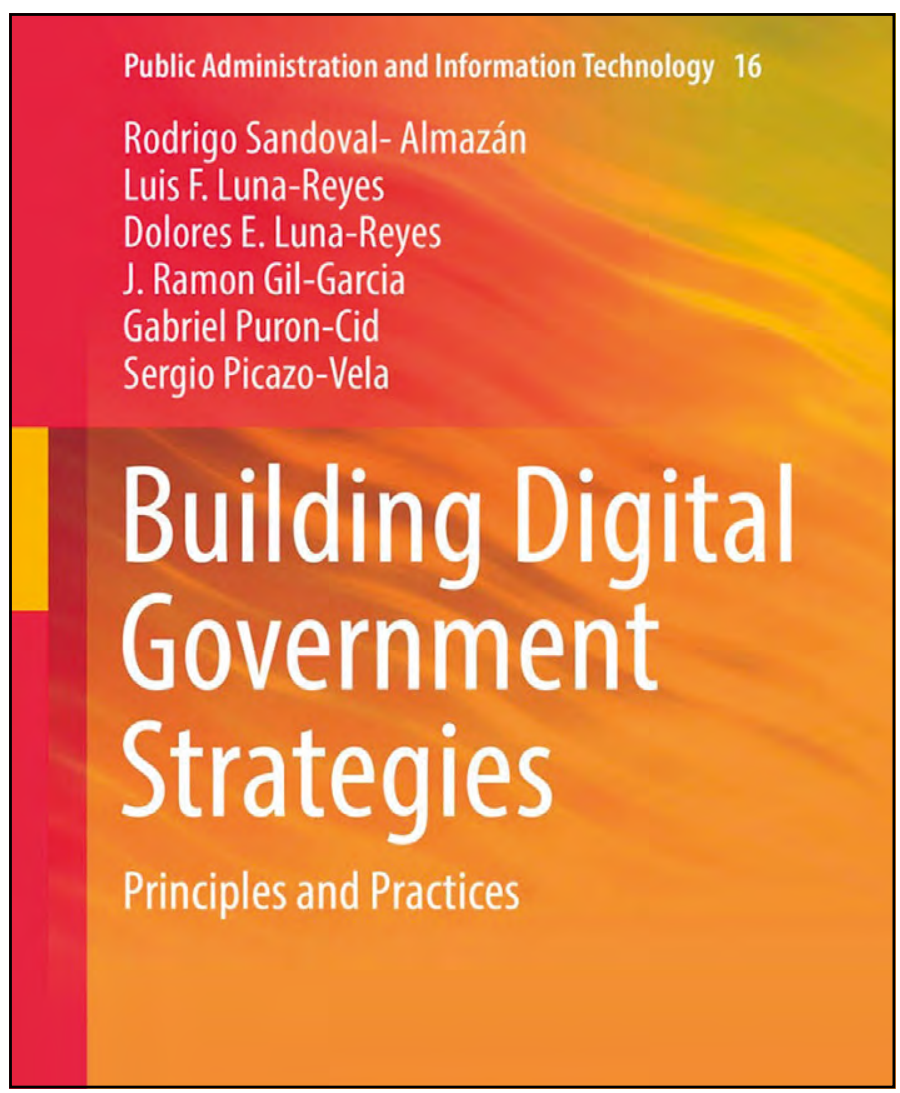

Portada del libro Building Digital Government Strategies.Principles and Practices [Construyendo estrategias de gobierno digital. Principios y prácticas]. 
IIVESTIGACIÓn Y CIERGIA DE LA UחIVERSIDAD AUTÓnOMH DE AGUASCALIERTES guía que proporciona principios generales y vías para entender y resolver los desafíos asociados con los proyectos y estrategias del gobierno digital. Se presenta una colección relevante de principios e ideas sobre estrategias para mejorar la implementación del gobierno digital.

En cada capítulo se presenta un pequeño caso de estudio para ilustrar el problema a resolver, describe los principales principios y presenta argumentos y conclusiones basadas en los hallazgos de la propia investigación y referencias de estudios previos. Este libro está estructurado en nueve capítulos y desarrolla siete áreas en las que se interrelacionan una serie de principios y estrategias: la primera se expone en el capítulo dos y se titula Desarrollando una Estrategia de Gobierno Digital para la Creación de Valor Público. Se definen los conceptos de estrategia digital gubernamental y empresarial. Considera que el diseño de la estrategia es un continuo proceso de negociación de planes y oportunidades para la creación de valor público, el cual es otro proceso de negociación y clarificación entre los actores del gobierno y otros grupos de interés.

La segunda área, descrita en el tercer capítulo, se refiere a la Conceptualización y Pre-planeación para un Proyecto de Gobierno Digital. Se argumenta que hay otros aspectos más allá de la tecnología que deben considerarse, la comprensión y la administración para incrementar la probabilidad de éxito de los proyectos para gobiernos digitales, incorporando múltiples dimensiones que incluyan aspectos sociales y técnicos. Se recomienda a los administradores públicos seguir una serie de pasos para entender mejor el problema, los grupos de interés y las soluciones potenciales de estos proyectos, se incluye también a los negocios.

La tercer área se describe en el cuarto capítulo, el Financiamiento y la Provisión de Recursos de las Estrategias del Gobierno Digital. Se proponen estrategias a lo largo del ciclo presupuestal del gobierno, se proporciona una guía para definir adecuadamente las fuentes de recursos y la implementación del presupuesto en proyectos del gobierno digital. Se identifican diversos principios para construir solidas estrategias de administración financiera para estos proyectos.
En la cuarta área, capítulo 5, Trabajo en Red del Gobierno y la Interoperabilidad de los sistemas, se introduce la idea del gobierno en red como una forma de organizar la entrega de servicios mediante la colaboración interorganizacional. El diseño institucional que enfatiza el proceso sobre la dirección y el control apoya la colaboración. El concepto de interoperabilidad combina aspectos técnicos con las políticas y otros aspectos organizacionales.

La quinta área se expone en el capítulo 6, denominado Administrando la Información Tecnológica en el Desarrollo de Proyectos. Aborda el desafío que tienen los gobiernos de generar metodologías para proyectos del gobierno digital con un diseño que reduzca el tiempo entre la concepción del proyecto y su implementación. Debido a los cambios tan rápidos de la información tecnológica se deben hacer ajustes de los planes en respuesta a los cambios del contexto con acciones ágiles y oportunas. Usar herramientas simples para que las organizaciones obtengan ventaja documentando sus experiencias sobre procesos, problemas, lecciones aprendidas y resultados en el desarrollo de proyectos de información tecnológica.

La sexta área, capítulo 7, Gobierno Abierto: Datos Abiertos y Proyectos, describe la importancia de ecosistemas de datos abiertos y su implementación en gobiernos abiertos. Se discute la importancia de una cultura de datos abiertos y la creación de comunidades que usen, compartan y colaboren con la información gubernamental. También se enfatiza la importancia de equipos de trabajo para crear oportunidades que promuevan la innovación al interior del gobierno abierto.

La séptima área, capitulo 8, Promoviendo el Compromiso Ciudadano, discute el rol de las herramientas electrónicas de doble vía, la comunicación y el compromiso entre las entidades gubernamentales y el público. Describe también el impacto social de la adopción de estas herramientas en la estructura organizacional del gobierno y sus beneficios para los ciudadanos. Se presentan recomendaciones para mejorar el éxito en las estrategias para la implementación de los diversos medios electrónicos.

Finalmente, en la conclusión del libro se hace una reflexión sobre estas siete áreas que presentan igual número de desafíos. 